\title{
MEASURES ON INDEPENDENT SETS, A QUANTITATIVE VERSION OF RUDIN'S THEOREM
}

\author{
T. W. KÖRNER
}

(Communicated by Michael T. Lacey)

\begin{abstract}
We construct measures with independent support whose Fourier coefficients decrease as fast as possible.
\end{abstract}

\section{INTRODUCTION}

We work on the circle $\mathbb{T}=\mathbb{R} / \mathbb{Z}$. Throughout this paper positive will mean strictly positive. We need the following definition.

Definition 1.1. A closed set $E$ is called independent if, given distinct points $x_{1}, x_{2}, \ldots, x_{q} \in E$, the only solution to the equation

$$
\sum_{j=1}^{q} m_{j} x_{j}=0
$$

with $m_{j} \in \mathbb{Z}$ is the trivial solution $m_{1}=m_{2}=\cdots=m_{q}=0$.

Lemma 1.2. Suppose that $\mu$ is a non-zero measure on $\mathbb{T}$ and $q$ is a positive integer such that we can find an $\alpha>1 / q$ and an $A>0$ with

$$
|\hat{\mu}(r)| \leq A|r|^{-\alpha}
$$

for all $r \neq 0$. Then we can find distinct points $x_{1}, x_{2}, \ldots, x_{q} \in \operatorname{supp} \mu$ and $m_{j} \in \mathbb{Z}$, not all zero, such that

$$
\sum_{j=1}^{q} m_{j} x_{j}=0 .
$$

Proof. Let $\mu_{q}=\mu * \mu * \cdots * \mu$, the convolution of $\mu$ with itself $q$ times. Then

$$
\left|\hat{\mu}_{q}(r)\right|=|\hat{\mu}(r)|^{q} \leq A^{q}|r|^{-q \alpha}
$$

for all $r \neq 0$. It follows that $\hat{\mu}_{q} \in l^{1}$ and so $d \mu_{q}(t)=f(t) d t$ for some continuous function $f$. Thus (since $\mu_{q}$ is non-zero) $\operatorname{supp} \mu_{q}$ contains a non-trivial interval and so a non-zero rational number $y$ and so we can find $y_{1}, y_{2}, \ldots, y_{q} \in \operatorname{supp} \mu$ such that

$$
\sum_{j=1}^{q} y_{j}=y .
$$

Received by the editors March 16, 2006.

2000 Mathematics Subject Classification. Primary 42A16.

Key words and phrases. Rudin set, independent set, Fourier series.

(C) 2007 American Mathematical Society Reverts to public domain 28 years from publication 
Since we do not know that the $y_{j}$ are distinct, we can only conclude that there exists a $q^{\prime}$ with $1 \leq q^{\prime} \leq q$ distinct points $x_{1}, x_{2}, \ldots, x_{q^{\prime}} \in \operatorname{supp} \mu$, and non-zero $n_{j} \in \mathbb{Z}$ such that

$$
\sum_{j=1}^{q^{\prime}} n_{j} x_{j}=y .
$$

If we take $n_{j}=0$ for $j>q^{\prime}$, it now follows that there are distinct points $x_{1}, x_{2}$, $\ldots, x_{q} \in \operatorname{supp} \mu$ and $n_{j} \in \mathbb{Z}$, not all zero, such that

$$
\sum_{j=1}^{q} n_{j} x_{j}=y .
$$

The stated result follows if we choose a non-zero $M \in \mathbb{Z}$ such that $M y=0$ and we set $m_{j}=M n_{j}$.

In the other direction, Rudin proved the following result (see [9]).

Theorem 1.3. There exists a probability measure $\mu$ such that $\hat{\mu}(r) \rightarrow 0$ as $|r| \rightarrow \infty$, but $\operatorname{supp} \mu$ is independent.

Since Rudin's original paper was published, several different constructions have been given by Kahane [2], Kaufman [6], and others.

In this paper we prove the following version of Rudin's result.

Theorem 1.4. Suppose that $\phi: \mathbb{N} \rightarrow \mathbb{R}$ is a sequence of positive numbers with $r^{\alpha} \phi(r) \rightarrow \infty$ as $r \rightarrow \infty$ whenever $\alpha>0$. Then there exists a probability measure $\mu$ such that $\phi(|r|) \geq|\hat{\mu}(r)|$ for all $r \neq 0$, but $\operatorname{supp} \mu$ is independent.

In view of Lemma 1.2, this result is best possible.

Our method of proof also proves the following theorem.

Theorem 1.5. If $q$ is an integer with $q \geq 1$ and $\psi: \mathbb{N} \rightarrow \mathbb{R}$ is a sequence of positive numbers such that $\psi(r) \rightarrow \infty$ as $r \rightarrow \infty$, then there exists a probability measure $\mu$ such that

$$
|\hat{\mu}(r)| \leq|r|^{-1 /(2 q)}(\log (1+|r|))^{1 / 2} \psi(|r|)
$$

for all $r \neq 0$, but, given distinct points $x_{1}, x_{2}, \ldots, x_{q} \in \operatorname{supp} \mu$, the only solution to the equation

$$
\sum_{j=1}^{q} m_{j} x_{j}=0
$$

with $m_{j} \in \mathbb{Z}$ is the trivial solution $m_{1}=m_{2}=\cdots=m_{q}=0$.

Although we believe that Theorems 1.4 and 1.5 are new (compare the results of Chapter 17 in [3]), the idea behind the proof comes from [7]. (The papers [10] and [9] use probabilistic ideas in a similar context and, to give another example, so does the proof of the maille condition on page 146 of [4].)

\section{The Basic COnstruction}

We shall need the following well-known theorem (see, for example Chapter 6 of $[3])$. 
Theorem 2.1. Suppose that $Z, Z_{1}, Z_{2}, \ldots, Z_{n}$ are independent identically distributed complex valued random variables with $|Z|=1$ and $\mathbb{E} Z=0$. Then

$$
\operatorname{Pr}\left(\left|\sum_{u=1}^{n} Z_{u}\right| \geq \lambda\right) \leq 4 \exp \left(-\lambda^{2} / n\right)
$$

for all $\lambda>0$.

The next lemma (with origins in [7]) forms the heart of this paper. We write $\delta_{t}$ for the unit Dirac point mass at $t$.

Lemma 2.2. Let $q$ be a positive integer and let $m_{1}, m_{2}, \ldots, m_{q}$ be non-zero integers. Then, provided only that $n$ is large enough, we can find distinct points $x_{1}$, $x_{2}, \ldots, x_{n}$ with the following two properties.

(i) If we write $\mu=n^{-1} \sum_{u=1}^{n} \delta_{x_{u}}$, we have

$$
|\hat{\mu}(r)| \leq 4 q^{1 / 2} n^{-1 / 2}(\log n)^{1 / 2}
$$

for all $1 \leq|r| \leq n^{4 q}$.

(ii) If $j(1), j(2), \ldots, j(q)$ are distinct integers with $1 \leq j(k) \leq n$, then

$$
\left|\sum_{k=1}^{q} m_{k} x_{j(k)}\right| \geq 8^{-1} n^{-q} .
$$

Proof. Consider the independent random variables $Y_{u}$ where each $Y_{u}$ is uniformly distributed over $\mathbb{T}$. We look at the random measure

$$
\sigma=n^{-1} \sum_{u=1}^{n} \delta_{Y_{u}}
$$

We note that

$$
\hat{\sigma}(r)=n^{-1} \sum_{u=1}^{n} \exp \left(2 \pi i r Y_{u}\right) .
$$

If $r \neq 0$, we see that the $\exp \left(2 \pi i r Y_{u}\right)$ are independent identically distributed complex valued random variables with $\left|\exp \left(2 \pi i r Y_{u}\right)\right|=1$ and $\mathbb{E} \exp \left(2 \pi i r Y_{u}\right)=0$. Thus, by Theorem 2.1, with $\lambda=4 q^{1 / 2} n^{1 / 2}(\log n)^{1 / 2}$,

$$
\begin{aligned}
& \operatorname{Pr}\left(|\hat{\sigma}(r)| \geq 4 q^{1 / 2} n^{-1 / 2}(\log n)^{1 / 2}\right) \\
& \quad=\operatorname{Pr}\left(\left|\sum_{u=1}^{n} \exp \left(2 \pi i r Y_{u}\right)\right| \geq 4 q^{1 / 2} n^{1 / 2}(\log n)^{1 / 2}\right) \\
& \quad \leq 4 \exp (-16 q \log n)=4 n^{-16 q} .
\end{aligned}
$$

Thus, provided only that $n$ is large enough,

$$
\begin{aligned}
\operatorname{Pr}(|\hat{\sigma}(r)| & \left.\geq 4 q^{1 / 2} n^{-1 / 2}(\log n)^{1 / 2} \text { for some } 1 \leq|r| \leq n^{4 q}\right) \\
& \leq \sum_{1 \leq|r| \leq n^{4 q}} \operatorname{Pr}\left(|\hat{\sigma}(r)| \geq 4 q^{1 / 2} n^{-1 / 2}(\log n)^{1 / 2}\right) \leq 2 n^{4 q} 4 n^{-16 q} \leq 1 / 4 .
\end{aligned}
$$


Now suppose that $j(1), j(2), \ldots, j(q)$ are distinct integers with $1 \leq j(k) \leq n$. By symmetry or direct calculation, the random variable

$$
\sum_{k=1}^{q} m_{k} Y_{j(k)}
$$

is uniformly distributed and so

$$
\operatorname{Pr}\left(\sum_{k=1}^{q} m_{k} Y_{j(k)} \in\left[-8^{-1} n^{-q}, 8^{-1} n^{-q}\right]\right)=4^{-1} n^{-q} .
$$

There are no more than $n^{q}$ different $q$-tuples $j(1), j(2), \ldots, j(q)$ of the type discussed. So, by the same kind of argument as we used in the previous paragraph, the probability that

$$
\sum_{k=1}^{q} m_{k} Y_{j(k)} \in\left[-8^{-1} n^{-q}, 8^{-1} n^{-q}\right]
$$

for any such $q$-tuple is no more than $1 / 4$.

Combining the results of our last two paragraphs, we see that, provided $n$ is large enough, the probability that $x_{u}=Y_{u}$ will fail to satisfy the conditions of our lemma is at most $1 / 2$. Since there must be an instance of any event with positive probability, the required result follows.

The next lemma merely serves to establish notation.

Lemma 2.3. Let $K: \mathbb{R} \rightarrow \mathbb{R}$ be an infinitely differentiable function with the following properties.

$\left(i^{\prime}\right) K(x) \geq 0$ for all $x \in \mathbb{R}$

(ii') $\int_{\mathbb{R}} K(x) d x=1$.

(iii') $K(x)=0$ for $|x| \geq 1 / 4$.

If $N$ is a positive integer and we define $K_{N}: \mathbb{T} \rightarrow \mathbb{R}$ by

$$
K_{N}(t)= \begin{cases}N K(N t) & \text { if }|t| \leq 1 /(4 N), \\ 0 & \text { otherwise, }\end{cases}
$$

then $K_{N}$ is an infinitely differentiable function having the following properties.

(i) $K_{N}(t) \geq 0$ for all $t \in \mathbb{T}$.

(ii) $\int_{\mathbb{T}} K_{N}(t) d t=1$.

(iii) $K_{N}(t)=0$ for $|t| \geq 1 /(4 N)$.

(iv) $\left|\hat{K}_{N}(r)\right| \leq 1$ for all $r$.

(v) There exists a constant $A$, independent of $N$, such that $\left|\hat{K}_{N}(r)\right| \leq A(N / r)^{2}$ for all $r \neq 0$.

Proof. This is entirely straightforward. To obtain (v) we integrate by parts to get

$$
\begin{aligned}
\left|\hat{K}_{N}(r)\right| & =\frac{1}{|r|^{2}}\left|\int_{\mathbb{T}} K_{N}^{(2)}(t) \exp (-2 \pi i r t) d t\right| \\
& \leq \frac{1}{|r|^{2}} \int_{\mathbb{T}}\left|K_{N}^{(2)}(t)\right| d t=\frac{N^{2}}{|r|^{2}} \int_{\mathbb{T}}\left|K^{(2)}(t)\right| d t .
\end{aligned}
$$

We now "spread out" the measure of Lemma 2.2 to obtain the measure used in our construction. 
Theorem 2.4. Suppose that $\psi: \mathbb{N} \rightarrow \mathbb{R}$ is a sequence of positive numbers such that $\psi(r) \rightarrow \infty$ as $r \rightarrow \infty$. Suppose that $q$ is a positive integer, $\epsilon, \delta>0$ and $\mathbf{m}=\left(m_{1}, m_{2}, \ldots, m_{q}\right) \in \mathbb{Z}^{q}$ with $M=\sum_{k=1}^{q}\left|m_{k}\right| \neq 0$. Then we can find an infinitely differentiable function $f: \mathbb{T} \rightarrow \mathbb{R}$ with the following properties.

(i) $f(t) \geq 0$ for all $t$.

(ii) $\int_{\mathbb{T}} f(t) d t=1$.

(iii) $|\hat{f}(r)| \leq \epsilon|r|^{-1 /(2 q)}(\log (1+|r|))^{1 / 2} \psi(|r|)$ for all $r \neq 0$.

(iv) If $t_{k} \in \operatorname{supp} f$ for $1 \leq k \leq q$ and $\left|t_{k}-t_{l}\right| \geq \delta$ for $1 \leq k<l \leq q$, then

$$
\sum_{k=1}^{q} m_{k} t_{k} \neq 0
$$

Proof. Provided only that $n$ is large enough, we can find $x_{u}$ and $\mu$ satisfying the conditions of Lemma 2.2.

Now take $N(n)=4 M n^{q}$, let $K_{N(n)}$ be defined as in Lemma 2.3, and set $f=$ $\mu * K_{N(n)}$. Conclusions (i) and (ii) are immediate.

Now suppose $n$ sufficiently large that $n^{4 q}>N(n)$ and $N(n) \geq \delta^{-1}$. If $t_{k} \in \operatorname{supp} f$ for $1 \leq k \leq q$ and $\left|t_{k}-t_{l}\right| \geq \delta$ for $1 \leq k<l \leq q$, then, since

$$
\operatorname{supp} f \subseteq \bigcup_{u=1}^{n}\left[x_{u}-N(n)^{-1} / 4, x_{u}+N(n)^{-1} / 4\right],
$$

it follows that we can find distinct integers $j(1), j(2), \ldots, j(q)$ with $1 \leq j(k) \leq n$ such that

$$
\left|x_{j(k)}-t_{k}\right| \leq N(n)^{-1} / 4
$$

for all $1 \leq k \leq q$. Condition (ii) of Lemma 2.2 now tells us that

$$
\begin{aligned}
\left|\sum_{k=1}^{q} m_{k} t_{k}\right| & \geq\left|\sum_{k=1}^{q} m_{k} x_{j(k)}\right|-\sum_{k=1}^{q}\left|m_{k}\right|\left|x_{j(k)}-t_{k}\right| \\
& \geq 8^{-1} n^{-q}-4^{-1} M N(n)^{-1}=16^{-1} n^{-q}>0
\end{aligned}
$$

and condition (iv) follows.

We bound $|\hat{f}(r)|$ using condition (i) of Lemma 2.2, Lemma 2.3, and the trivial bounds $|\hat{f}(r)|,|\hat{\mu}(r)| \leq 1$. If $1 \leq|r| \leq N(n)$, then

$$
|\hat{f}(r)| \leq|\hat{\mu}(r)| \leq 4 q^{1 / 2} n^{-1 / 2}(\log n)^{1 / 2} \leq C_{1} N(n)^{-1 /(2 q)}(\log N(n))^{1 / 2}
$$

for some constant $C_{1}$ independent of $n$. If $N(n) \leq|r| \leq n^{4 q}$, then

$$
\begin{aligned}
|\hat{f}(r)| & \leq|\hat{\mu}(r)|\left|\hat{K}_{N(n)}(r)\right| \leq\left(4 q^{1 / 2} n^{-1 / 2}(\log n)^{1 / 2}\right)\left(A(N(n) / r)^{2}\right) \\
& \leq C_{2} N(n)^{-1 /(2 q)}(\log N(n))^{1 / 2}(N(n) / r)^{2} \\
& =C_{2}(N(n) / r)^{2-1 /(2 q)} r^{-(1 / 2 q)}(\log N(n))^{1 / 2} \\
& \leq C_{3}|r|^{-1 /(2 q)}(\log |r|)^{1 / 2}
\end{aligned}
$$

for some constants $C_{2}$ and $C_{3}$ independent of $n$. If $|r| \geq n^{4 q}$, then

$$
|\hat{f}(r)| \leq\left|\hat{K}_{N(n)}(r)\right| \leq A(N(n) / r)^{2}=A|r|^{-1}\left(N(n)^{2} /|r|\right) \leq C_{4}|r|^{-1 /(2 q)}(\log |r|)^{1 / 2}
$$

for some constant $C_{4}$ independent of $n$.

Since $\psi(r) \rightarrow \infty$ as $r \rightarrow \infty$, it follows that, provided only that $n$ is large enough,

$$
|\hat{f}(r)| \leq \epsilon|r|^{-1 /(2 q)}(\log (1+|r|))^{1 / 2} \psi(|r|)
$$

for all $r \neq 0$ and condition (iv) holds. 
The method of proof used in the next section requires us to make a further simple observation.

Lemma 2.5. Given $\epsilon>0$, we can find an $\eta>0$ such that, if $\mu$ is a probability measure with $|\hat{\mu}(r)| \leq \eta$ for $r \neq 0$, we know that $\operatorname{supp} \mu$ intersects every interval of length $\epsilon$.

Proof. By translation, it suffices to show that $\operatorname{supp} \mu$ intersects $(-\epsilon / 2, \epsilon / 2)$. Choose an integer $N$ with $N \geq \epsilon^{-1}$. If $\operatorname{supp} \mu$ does not intersect $(-\epsilon / 2, \epsilon / 2)$, then

$$
\begin{aligned}
0= & \left|\int_{\mathbb{T}} K_{N}(t) d \mu(t)\right|=\left|\sum_{r=-\infty}^{\infty} \hat{K}_{N}(-r) \hat{\mu}(r)\right| \\
& \geq\left|\hat{K}_{N}(0)\right||\hat{\mu}(0)|-\sum_{r \neq 0}\left|\hat{K}_{N}(-r) \hat{\mu}(r)\right| \geq 1-2 \eta A_{2} N^{2} \sum_{r=1}^{\infty} r^{-2},
\end{aligned}
$$

which is impossible if $\eta$ is sufficiently small.

Theorem 2.6. Theorem 2.4 holds with the additional condition:

(v) If $I$ is an interval of length $\epsilon$, then $\operatorname{supp} f \cap I \neq \emptyset$.

\section{Proof OF THE MAIN THEOREM}

We prove Theorem 1.4 by using a Baire category argument. (The reader will not need to be reminded that the introduction of Baire category methods to harmonic analysis is also due to Kaufman [5].) Like most Baire category constructions this can be replaced by a direct construction using careful bookkeeping. However, the author discovered Theorem 1.4 in the course of seeking a Baire category proof of Rudin's original result, and it seems reasonable to present it in this way.

Any Baire category argument requires an appropriate complete metric space.

Lemma 3.1. (i) Consider the space $\mathcal{F}$ of non-empty closed subsets of $\mathbb{T}$. If we set

$$
d_{\mathcal{F}}(E, F)=\sup _{e \in E} \inf _{f \in F}|e-f|+\sup _{f \in F} \inf _{e \in E}|e-f|,
$$

then $\left(\mathcal{F}, d_{\mathcal{F}}\right)$ is a complete metric space.

(ii) Suppose $\phi: \mathbb{N} \rightarrow \mathbb{R}$ is a positive sequence. Then the space of sequences $\mathbf{a}: \mathbb{Z} \rightarrow \mathbb{C}$ with $\sup _{r \in \mathbb{Z}} \phi(|r|)^{-1}\left|a_{r}\right|$ finite is a complete normed space under the norm

$$
\|\mathbf{a}\|_{\phi}=\sup _{r \in \mathbb{Z}} \phi(|r|)^{-1}\left|a_{r}\right| .
$$

(iii) Consider the space $\mathcal{P}_{\phi}$ consisting of ordered pairs $(E, \mu)$ where $E$ is a closed subset of $\mathbb{T}$ and $\mu$ is a probability measure with $\operatorname{supp} \mu \subseteq E$ and $\sup _{r \in \mathbb{Z}} \phi(|r|)^{-1}|\hat{\mu}(r)|$ finite. Then

$$
d_{\phi}((E, \mu),(F, \sigma))=d(E, F)+\|\hat{\mu}-\hat{\sigma}\|_{\phi}
$$

is a complete metric on $\mathcal{P}_{\phi}$.

(iv) If

$$
\mathcal{G}_{\phi}=\left\{(E, \mu) \in \mathcal{P}_{\phi}: \phi(|r|)^{-1}|\hat{\mu}(r)| \rightarrow 0 \text { as }|r| \rightarrow \infty\right\},
$$

then $\mathcal{G}_{\phi}$ is a non-empty closed subset of $\mathcal{P}_{\phi}$. 
Proof. (i) The metric $d$ is called the Hausdorff metric and is introduced in [1]. Its properties are derived (usually as an exercise) in many textbooks.

(ii) Standard.

(iii) Use properties of weak convergence of measures.

(iv) Standard. Observe that, if $m$ is a Lebesgue measure, $(\mathbb{T}, m) \in \mathcal{G}_{\phi}$.

We use the fact $\left(\mathcal{G}_{\phi}, d_{\phi}\right)$ is a non-empty complete metric space to obtain a Baire category version of Theorem 1.4 .

Theorem 3.2. Suppose that $\phi: \mathbb{N} \rightarrow \mathbb{R}$ is such that $\phi(0)=1, \phi(r)>0$ for all $r$, and $r^{\alpha} \phi(r) \rightarrow \infty$ as $r \rightarrow \infty$ whenever $\alpha>0$. Then the set of $(E, \mu) \in \mathcal{G}_{\phi}$ such that $E$ has a complement of first category in $\left(\mathcal{G}_{\phi}, d_{\phi}\right)$.

We obtain Theorem 3.2 by studying the set $\mathcal{H}(q, p, \mathbf{m})$ defined as follows.

Definition 3.3. Suppose that $\phi$ is as in Theorem 3.2, $q$ and $p$ are positive integers, and $\mathbf{m}=\left(m_{1}, m_{2}, \ldots, m_{q}\right) \in \mathbb{Z}^{q}$ with

$$
M=\sum_{j=1}^{q}\left|m_{j}\right| \neq 0 .
$$

Then the set $\mathcal{H}(q, p, \mathbf{m})$ consists of those $(E, \mu) \in \mathcal{G}_{\phi}$ such that $\sum_{j=1}^{q} m_{j} x_{j} \neq 0$ whenever $x_{j} \in E$ and $\left|x_{i}-x_{j}\right| \geq 1 / p$ for $i \neq j$.

Since the set of finite sequences of integers is countable, Theorem 3.2 follows from the following lemma.

Lemma 3.4. The set $\mathcal{H}(q, p, \mathbf{m})$ is open and dense in $\left(\mathcal{G}_{\phi}, d_{\phi}\right)$.

We split the proof of Lemma 3.4 into two parts.

Lemma 3.5. The set $\mathcal{H}(q, p, \mathbf{m})$ is open.

Proof. We show that the complement of $\mathcal{H}(q, p, \mathbf{m})$ is closed. Suppose that $\left(E_{r}, \mu_{r}\right)$ $\notin \mathcal{H}(q, p, \mathbf{m})$ and $\left(E_{r}, \mu_{r}\right) \rightarrow(E, \mu)$. We can find $x_{j}(r) \in E_{r}$ such that $\mid x_{i}(r)-$ $x_{j}(r) \mid \geq 1 / p$ for $i \neq j$ and

$$
\sum_{j=1}^{q} m_{j} x_{j}(r)=0 .
$$

By an appropriate form of the Bolzano-Weierstrass theorem, we can find $x_{j} \in \mathbb{T}$ and $r(k) \rightarrow \infty$ such that $x_{j}(r(k)) \rightarrow x_{j}$ for each $1 \leq j \leq q$. Automatically, $\left|x_{i}-x_{j}\right| \geq 1 / p$ for $i \neq j$ and

$$
\sum_{j=1}^{q} m_{j} x_{j}=0 .
$$

Since $d_{\mathcal{F}}\left(E_{r(k)}, E\right) \rightarrow 0$ it follows that $x_{j} \in E$ for $1 \leq j \leq q$ and so $(E, \mu) \notin$ $\mathcal{H}(q, p, \mathbf{m})$ as required.

Our proof of the remaining part of Lemma 3.4 uses the following two simple observations.

Lemma 3.6. Given $(E, \mu) \in \mathcal{G}_{\phi}$ and $\epsilon>0$ we can find an $(F, \sigma) \in \mathcal{G}_{\phi}$ with $d_{\phi}((E, \mu),(F, \sigma))<\epsilon$ having the following properties.

(i) $d \sigma(x)=g(x) d m(x)$, where $g$ is infinitely differentiable and $m$ is a Lebesgue measure. 
(ii) There exists an $\alpha>0$ such that, whenever $x \in F$, we can find an interval $I=[y-\alpha, y+\alpha]$ such that $x \in I \subseteq F$.

Proof. Choose $u_{n}: \mathbb{T} \rightarrow \mathbb{R}$, a non-negative, infinitely differentiable function, such that $\operatorname{supp} u_{n} \subseteq[-1 / n, 1 / n]$ and $\int_{\mathbb{T}} u_{n}(t) d t=1$. Provided that $n$ is large enough, standard theorems show that $g=u_{n} * \sigma, d \sigma(x)=g(x) d m(x)$, and $F=E+$ $[-1 / n, 1 / n]$ satisfy the conclusions of the lemma.

Lemma 3.7. There exists a constant $A$ with the following property. Suppose that $\omega: \mathbb{N} \rightarrow \mathbb{R}$ is a sequence of positive numbers with $\omega(0)=1$,

$$
K^{-1} n^{-1} \leq \omega(n)
$$

for all $n \neq 0$, and

$$
K^{-1} \omega(n) \leq \omega(r) \leq K \omega(n)
$$

for all $1 \leq n \leq r \leq 2 n$ and some constant $K>1$. Suppose also that $f$ and $g$ are continuous functions with $\hat{f}(0)=1$ and

$$
|\hat{g}(r)| \leq B r^{-2}, \quad|\hat{f}(r)| \leq C \omega(|r|)
$$

for all $r \neq 0$. Then

$$
|\widehat{f \times g}(r)-\hat{g}(r)| \leq A B C K \omega(r)
$$

for all $r$.

Proof. We have

$$
\begin{aligned}
|\widehat{f \times g}(r)-\hat{g}(r)| & =\left|\sum_{u \neq 0} \hat{f}(r-u) \hat{g}(u)\right| \leq \sum_{u \neq 0}|\hat{f}(r-u) \hat{g}(u)| \\
& \leq B C \sum_{u \neq 0} \frac{\omega(|r-u|)}{u^{2}} \\
& =B C \sum_{0<|u| \leq|r| / 2} \frac{\omega(|r-u|)}{u^{2}}+B C \sum_{|u|>r / 2} \frac{\omega(|r-u|)}{u^{2}} \\
& \leq B C \sum_{|u| \leq|r| / 2} \frac{K \omega(|r|)}{u^{2}}+B C \sum_{|u|>|r| / 2} \frac{1}{u^{2}} \\
& \leq A_{1} B C K \omega(|r|)+A_{2} B C \frac{1}{|r|} \leq A B C K \omega(|r|)
\end{aligned}
$$

for appropriate constants $A_{1}, A_{2}$, and $A$ and all $r \neq 0$. A similar calculation works for $r=0$.

We can now complete the proof of Lemma 3.4.

Lemma 3.8. The set $\mathcal{H}(q, p, \mathbf{m})$ is dense in $\left(\mathcal{G}_{\phi}, d_{\phi}\right)$.

Proof. We wish to show that, given any $1 / 10>\eta>0$ and any $(E, \mu) \in \mathcal{G}_{\phi}$, we can find an $(F, \sigma) \in \mathcal{H}(q, p, \mathbf{m})$ with

$$
d_{\phi}((E, \mu),(F, \sigma))<\eta .
$$

In view of Lemma 3.6, we may suppose that $d \mu(x)=g(x) d m(x)$, where $g$ is infinitely differentiable and there exists an $\alpha>0$ such that every point of supp $g$ lies in an 
interval $I \subseteq \operatorname{supp} g$ of length at least $\alpha>0$. Thus there exists a constant $B$ such that

for all $r \neq 0$.

$$
|\hat{g}(r)| \leq B|r|^{-2}
$$

Theorem 2.6 tells us that, if $\epsilon>0$, we can find an infinitely differentiable function $f_{\epsilon}: \mathbb{T} \rightarrow \mathbb{R}$ with the following properties.

(i) $f_{\epsilon}(t) \geq 0$ for all $t$.

(ii) $\int_{\mathbb{T}} f_{\epsilon}(t) d t=1$.

(iii) $\left|\hat{f}_{\epsilon}(r)\right| \leq \epsilon|r|^{-1 /(4 q)}$ for all $r \neq 0$.

(iv) If $x_{j} \in \operatorname{supp} f_{\epsilon}$ for $1 \leq j \leq q$, and $\left|x_{j}-x_{k}\right| \geq 1 / p$ for $1 \leq j<k \leq q$, then

$$
\sum_{j=1}^{q} m_{j} x_{j} \neq 0 .
$$

(v) If $I$ is an interval of length $\epsilon$, then supp $f_{\epsilon} \cap I \neq \emptyset$.

If we set $g_{\epsilon}(t)=g(t) f_{\epsilon}(t)$, and $E_{\epsilon}=E \cap \operatorname{supp} f_{\epsilon}$, then, automatically

$\left(\mathrm{i}^{\prime}\right) f_{\epsilon}(t) \geq 0$ for all $t$,

and, since $\operatorname{supp} g_{\epsilon}=\operatorname{supp} f_{\epsilon} \cap \operatorname{supp} g$, it follows that supp $g_{\epsilon} \subseteq E_{\epsilon}$, and

$\left(\right.$ iv $\left.^{\prime}\right)$ if $x_{j} \in \operatorname{supp} g_{\epsilon}$ for $1 \leq j \leq q$, and $\left|x_{j}-x_{k}\right| \geq 1 / p$ for $1 \leq j<k \leq q$, then

$$
\sum_{j=1}^{q} m_{j} x_{j} \neq 0 .
$$

On the other hand, condition (v) tells us that, provided only $\epsilon$ is small enough,

$$
d\left(E_{\epsilon}, E\right)<\eta / 2 \text {. }
$$

If we take $\omega(0)=1, \omega(r)=r^{-1 / 4 q}$ for $r \geq 1$ and $C=\epsilon$ in Lemma 3.7, the inequality proved there shows that, if $\gamma>0$ is fixed, $\left|\hat{g}_{\epsilon}(0)-\hat{g}(0)\right| \leq \gamma$ and

$$
\left|\hat{g}_{\epsilon}(r)-\hat{g}(r)\right| \leq \gamma r^{-1 / 4 q}
$$

for all $r \neq 0$ and all sufficiently small $\epsilon$. Since $r^{1 / 4 q} \phi(r) \rightarrow \infty$ as $r \rightarrow \infty$, it follows that, if $\beta>0$ is fixed with $1 / 10>\beta>0$,

$$
\left\|\hat{g}_{\epsilon}-\hat{g}\right\|_{\phi}<\beta
$$

for all $\epsilon$ sufficiently small. In particular, we know that

$$
\left|\hat{g}_{\epsilon}(0)-1\right|=\left|\hat{g}_{\epsilon}(0)-\hat{g}(0)\right|<\beta
$$

for $\epsilon$ sufficiently small. Writing $G_{\epsilon}=\hat{g}_{\epsilon}(0)^{-1} g_{\epsilon}$, we have

$$
\begin{aligned}
\left\|\hat{G}_{\epsilon}-\hat{g}\right\|_{\phi} & =\left\|\frac{\hat{g}_{\epsilon}}{\hat{g}(0)}-\hat{g}\right\|_{\phi} \\
& \leq\left\|\hat{g}_{\epsilon}-\hat{g}\right\|_{\phi}+\left(1-\frac{1}{\hat{g}(0)}\right)\left(\|\hat{g}\|_{\phi}+\left\|\hat{g}_{\epsilon}-\hat{g}\right\|_{\phi}\right) \\
& \leq \beta+2 \beta\left(\|\hat{g}\|_{\phi}+\beta\right) .
\end{aligned}
$$

It follows that $G_{\epsilon} \in \mathcal{G}_{\phi}$ and, provided only that $\beta$ (and so $\epsilon$ ) is small enough,

$$
\left\|\hat{G}_{\epsilon}-\hat{g}\right\|_{\phi}<\eta / 2 \text {. }
$$

Thus, provided only that $\epsilon$ is small enough, $F=E_{\epsilon}$ and $d \sigma(x)=G_{\epsilon}(x) d m(x)$ satisfy the conclusions required by the first sentence of this proof. 
We have thus proved Theorem 3.2 and so Theorem 1.4. We can prove Lemma 1.5 in the same way, obtaining the following Baire category version.

Lemma 3.9. Suppose $q$ is an integer with $q \geq 1$ and $\psi: \mathbb{N} \rightarrow \mathbb{R}$ is a sequence of strictly positive numbers with $\psi(r) \rightarrow \infty$ as $r \rightarrow \infty$. Then, if we write $\phi(r)=$ $r^{-1 /(2 q)}(\log (1+|r|))^{1 / 2} \psi(|r|)$ for $r \geq 1$ and set $\phi(0)=1$, the set of $(E, \mu) \in \mathcal{G}_{\phi}$ with the property that, given distinct points $x_{1}, x_{2}, \ldots, x_{q} \in E$, the only solution to the equation

$$
\sum_{j=1}^{q} m_{j} x_{j}=0
$$

with the $m_{j} \in \mathbb{Z}$ is the trivial solution $m_{1}=m_{2}=\cdots=m_{q}=0$, has a complement of first category in $\left(\mathcal{G}_{\phi}, d_{\phi}\right)$.

There is large gap between the results of Lemma 1.2 and Theorem 1.5. When $q=1$ we can improve Theorem 1.5 using the arguments of [8]. These show that, if $\omega: \mathbb{N} \rightarrow \mathbb{R}$ is a sequence of positive numbers with $\sum_{r=1}^{\infty} \omega(r)$ divergent and $\omega$ satisfies certain regularity conditions, then we can find a probability measure $\mu$ with $|\hat{\mu}(r)| \leq \omega(r)$ for all $r \neq 0$ whose support contains no rational number.

I should like to thank the referee for saving me from a very serious error.

\section{REFERENCES}

1. F. Hausdorff, Set theory. Second edition. Translated from the German by John R. Aumann et al., Chelsea Publishing Co., New York, 1957. MR0086020 (19:111a)

2. J. P. Kahane, Images d'ensembles parfaits par des séries de Fourier gaussienes C. R. Acad. Sci. Paris Sér. A-B 263 (1966) A678-A681.

3. J. P. Kahane, Some random series of functions. Second edition. Cambridge Studies in Advanced Mathematics, 5. Cambridge University Press, Cambridge, 1985. MR833073 (87m:60119)

4. J. P. Kahane and R. Salem, Ensembles parfaits et séries trigonométriques. Herman, Paris, 1963. MR0160065 (28:3279)

5. R. Kaufman, A functional method for linear sets Israel J. Math. 5 (1967) 185-7. MR0236607 (38:4902)

6. R. Kaufman, Measures in independent sets Studia Math. 30 (1968) 17-20. MR0227698 (37:3282)

7. R. Kaufman, Small subsets of finite Abelian groups Annales de l'Institut Fourier 18 (1968) 99-102. MR0241532 (39:2872)

8. T. W. Körner A topological Ivašev-Musatov theorem J. London Math. Soc. (2) 67 (2003), 448-460 MR1956146 (2004b:42006)

9. W. Rudin Fourier-Stieltjes transforms of measures on independent sets Bull. Amer. Math. Soc. 66 (1960) 199-202. MR0119035 (22:9802)

10. R. Salem, On singular monotonic functions whose spectrum has a given Hausdorff dimension Ark. Mat. 1 (1951) 353-365. MR0043249 (13:230b)

DPMms, Centre for Mathematical Sciences, Clarkson Road, Cambridge, England

E-mail address: twk@dpmms.cam.ac.uk 